\title{
Perineuronal Nets Suppress Plasticity of Excitatory Synapses on CA2 Pyramidal Neurons
}

\author{
Kelly E. Carstens, ${ }^{1,2}$ Mary L. Phillips, ${ }^{3} \mathbb{C}^{-}$Lucas Pozzo-Miller, ${ }^{3}$ Richard J. Weinberg, ${ }^{2}$ and ${ }^{\oplus S e r e n a ~ M . ~ D u d e k ~}{ }^{1}$ \\ ${ }^{1}$ Neurobiology Laboratory, National Institute of Environmental Health Sciences, National Institutes of Health, Research Triangle Park, North Carolina \\ 27709, ${ }^{2}$ Department of Cell Biology and Physiology, University of North Carolina, Chapel Hill, North Carolina 27599, and ${ }^{3}$ Department of Neurobiology, \\ Civitan International Research Center, The University of Alabama at Birmingham, Birmingham, Alabama 35294
}

\begin{abstract}
Long-term potentiation of excitatory synapses on pyramidal neurons in the stratum radiatum rarely occurs in hippocampal area CA2. Here, we present evidence that perineuronal nets (PNNs), a specialized extracellular matrix typically localized around inhibitory neurons, also surround mouse CA2 pyramidal neurons and envelop their excitatory synapses. CA2 pyramidal neurons express mRNA transcripts for the major PNN component aggrecan, identifying these neurons as a novel source for PNNs in the hippocampus. We also found that disruption of PNNs allows synaptic potentiation of normally plasticity-resistant excitatory CA2 synapses; thus, PNNs play a role in restricting synaptic plasticity in area CA2. Finally, we found that postnatal development of PNNs on CA2 pyramidal neurons is modified by early-life enrichment, suggesting that the development of circuits containing CA2 excitatory synapses are sensitive to manipulations of the rearing environment.
\end{abstract}

Key words: critical period; extracellular matrix; hippocampus; long-term potentiation

\section{Significance Statement}

Perineuronal nets (PNNs) are thought to play a major role in restricting synaptic plasticity during postnatal development, and are altered in several models of neurodevelopmental disorders, such as schizophrenia and Rett syndrome. Although PNNs have been predominantly studied in association with inhibitory neurons throughout the brain, we describe a dense expression of PNNs around excitatory pyramidal neurons in hippocampal area CA2. We also provide insight into a previously unrecognized role for PNNs in restricting plasticity at excitatory synapses and raise the possibility of an early critical period of hippocampal plasticity that may ultimately reveal a key mechanism underlying learning and memory impairments of PNN-associated neurodevelopmental disorders.

\section{Introduction}

Although components of the extracellular matrix (ECM) have been implicated in promoting synaptic plasticity, perineuronal nets (PNNs), a specialized form of ECM typically found around inhibitory neurons, are thought to inhibit plasticity (Dityatev et al., 2010). We previously identified hippocampal area CA2 as a region where synaptic plasticity is limited, even relatively early in

Received Jan. 13, 2016; revised April 6, 2016; accepted May 2, 2016.

Author contributions: K.E.C., L.P.-M., R.J.W., and S.M.D. designed research; K.E.C., M.L.P., and R.J.W. performed research; K.E.C. analyzed data; K.E.C., L.P.-M., R.J.W., and S.M.D. wrote the paper.

This work was supported by the Intramural Research Program of the National Institute of Environmental Health Sciences, National Institutes of Health (Z01 ES100221); by National Institute of Neurological Disorders and Stroke Award R01 NS039444 (to R.J.W.); and by National Institutes of Health Grant NS-065027 (to L.P.-M.). We thank Susan Burette for technical assistance with electron microscopy, the National Institute of Environmental Health Sciences Fluorescence Microscopy and Imaging Center and the animal care staff for support, and David Armstrong and Georgia Alexander for input on this manuscript.

The authors declare no competing financial interests.

Correspondence should be addressed to Serena M. Dudek at the above address. E-mail: dudek@niehs.nih.gov. DOI:10.1523/JNEUROSCI.0245-16.2016

Copyright $\odot 2016$ the authors $\quad 0270-6474 / 16 / 366312-09 \$ 15.00 / 0$ postnatal development (Zhao et al., 2007). Unlike PNNs in other brain regions, PNNs in area CA2 appear to be near excitatory synapses of pyramidal neurons (Celio, 1993; Fuxe et al., 1997; Costa et al., 2007), leading us to wonder whether PNNs are related to pyramidal neurons in some way.

PNNs first appear in the brain around postnatal day (PN) 14 in the mouse, and gradually increase until they are fully expressed in adulthood, often tracking the end of critical windows of synaptic plasticity (Hensch, 2004; Horii-Hayashi et al., 2015). Interestingly, the onset of PNN expression requires normal early experience in several brain regions, including motor, visual, and somatosensory systems, and similar manipulations performed in adulthood were without effect (Kalb and Hockfield, 1988; Guimarães et al., 1990; Kind et al., 1995; Lander et al., 1997; McRae et al., 2007). Because PNN deposition is experiencedependent and increases during postnatal development, these structures have been hypothesized to function in dampening synaptic plasticity during the closure of critical periods. For example, Pizzorusso et al. (2002) demonstrated an association between the 
presence and absence of PNNs in the visual cortex and the closure and reopening of ocular dominance plasticity, strongly supporting a role for PNNs in limiting experience-dependent plasticity. Because PNNs associated with pyramidal (i.e., presumed excitatory) neurons are rare in cortical structures (Alpár et al., 2006), the role of this matrix in limiting plasticity is widely thought to be due to their association with nonpyramidal (i.e., presumed inhibitory) neurons, specifically parvalbumin (PV)positive interneurons.

The structural and physiological effects of removing PNNs enzymatically have been well studied in inhibitory neurons. Degradation of PNNs around inhibitory neurons in culture (mainly PV-positive interneurons) with the bacterial enzyme chondroitinase (ChABC) increases interneuron excitability without affecting the number or distribution of perisomatic GABAergic presynaptic terminals (Dityatev et al., 2007), suggesting that PNNs function to regulate inhibitory neuron activity. Interestingly, manipulation of neuronal activity seems to modulate the development of PNNs both in culture and in vivo. For example, blocking neuronal activity in culture decreases PNNs around inhibitory neurons. Similarly, decreasing activity via dark rearing delays and attenuates PNN development around inhibitory neurons in the visual cortex (Pizzorusso et al., 2002).

Hippocampal area CA2, positioned between areas CA3 and CA1, has recently been appreciated as an important module of the hippocampus that is molecularly distinct from its neighboring areas (Caruana et al., 2012; Dudek et al., 2016). CA2 neurons receive excitatory synapses from the dentate gyrus, entorhinal cortex, and area CA3, and it is these synapses from CA3 in CA2 stratum radiatum (SR) that are highly resistant to plasticity (Zhao et al., 2007; Chevaleyre and Siegelbaum, 2010; Kohara et al., 2014). Although we have yet to fully understand the mechanism(s) behind this resistance to synaptic plasticity, several genes highly expressed in area CA2 are implicated in this lack of plasticity (Boulanger et al., 1995; Pelkey et al., 2002; Simons et al., 2009; Lee et al., 2010). Based on these observations and previous studies implicating PNNs in restricting plasticity, we investigated whether PNNs around pyramidal neurons in area CA2 play a role in restricting synaptic plasticity of CA2 excitatory synapses and are modified by early-life experience.

\section{Materials and Methods}

Animals. Animals in all experiments were housed under a 12:12 light/ dark cycle with access to food and water ad libitum. All procedures were approved by National Institute of Environmental Health Sciences, University of Alabama at Birmingham, and University of North Carolina Animal Care and Use Committees and were in accordance with the National Institutes of Health guidelines for care and use of animals.

Immunohistochemistry. Mouse lines expressing enhanced green fluorescent protein (EGFP) were used to label hippocampal CA2 pyramidal neurons (Gene Expression Nervous System Atlas, Amigo2-EGFP; Tg(Amigo2-EGFP)LW244Gsat/Mmcd) and GABAergic interneurons (Riken BioResource Center, Gad67-GFP; Cg-Gad1<tm1.1Tama $>$ ). Adult male mice were deeply anesthetized with Fatal-Plus and perfused with cold PBS, followed by $4 \%$ paraformaldehyde in PBS, pH 7.4. Brains were removed and postfixed overnight at $4^{\circ} \mathrm{C}$ and submerged in $30 \%$ sucrose. Forty-micrometer-thick sections were cut on a sliding microtome, blocked in 5\% normal goat serum and incubated in biotin-conjugated Wisteria floribunda agglutinin (WFA) lectin (1:1000; Sigma-Aldrich L1516) or antibodies anti-aggrecan (1:500; Millipore AB1031) or antineurocan (1:200; R\&D Systems AF5800) overnight at $4^{\circ} \mathrm{C}$. Sections were washed three times in PBS and incubated in secondary antibody at 1:500 for $40 \mathrm{~min}$ at room temperature: streptavidin Alexa-568 (Invitrogen), goat anti-rabbit $\mathrm{H}+\mathrm{L}$ A568 (Invitrogen), or goat anti-chicken $\mathrm{H}+\mathrm{L}$ (Invitrogen). Alternatively, the biotin-conjugated WFA lectin was amplified with the Vectastain Elite ABC kit (Vector Laboratories PK-6100) and reacted with 3,3'-diaminobenzidine (DAB) substrate kit (Vector Laboratories SK-4100). Sections were mounted with Vectashield antifade mounting medium with DAPI (Vector Laboratories). Images were acquired on a Zeiss laser scanning confocal (LSM510 NLO) or a Zeiss light microscope using controlled camera settings.

Immunohistochemistry and confocal microscopy of excitatory synapses on dendritic spines. Adult male mice of the thyl-GFP line M (7-20 months; Feng et al., 2000) were anesthetized with a mixture of ketamine and xylazine, and perfused as described above. Thirty-micrometer-thick coronal sections of the brain at the level of the dorsal hippocampus were made with a vibratome, and immunohistochemistry was performed on free-floating sections at room temperature. Sections were blocked and permeabilized by incubation in $10 \%$ goat serum albumin, $2 \%$ bovine serum albumin, and $0.4 \%$ Triton-X in PBS for $1 \mathrm{~h}$. Sections were incubated with biotin-WFA (1:100) and primary antibodies diluted in 5\% goat serum albumin, $2 \%$ bovine serum albumin, and $0.1 \%$ Triton- $\mathrm{X}$ in PBS for approximately 48 hours. Primary antibodies used were guinea pig anti-VGLUT1 (1:1000; EMD Millipore AB5905) and rabbit anti-GFP (1:2000; Abcam 290). After washing three times for $5 \mathrm{~min}$ in PBS, sections were incubated for $4 \mathrm{~h}$ in Streptavidin-594 (1:1000; Life Technologies) and fluorescently labeled secondary antibodies (anti-rabbit Alexa488, 1:500; Jackson ImmunoResearch; anti-guinea pig Alexa-647, 1:500; Jackson ImmunoResearch). Sections were washed three times for $5 \mathrm{~min}$ in PBS, incubated with DAPI ( $300 \mathrm{~nm}$ ) for $5 \mathrm{~min}$, and washed with PBS for 5 min before mounting with Vectashield antifade mounting media (Vector Laboratories). Sections were imaged in a laser-scanning confocal microscope (Zeiss Spectral LSM510-META) equipped with a multiline argon laser (458, 477, 488, and $514 \mathrm{~nm}$ ) and two HeNe lasers (543 and $633 \mathrm{~nm}$ ) using an oil-immersion $100 \times$ objective (1.4 numerical aperture). Image stacks through the $z$ plane were acquired at $0.1 \mu \mathrm{m}$, and displayed as maximum-intensity projections in ImageJ software (National Institutes of Health).

Electron microscopy. Adult mice (Charles River) were perfused with $4 \%$ paraformaldehyde (0.1 м phosphate buffer, $\mathrm{pH} 7.2)$ and $0.1 \%$ glutaraldehyde for $10 \mathrm{~min}$. Brains were postfixed at $4^{\circ} \mathrm{C}$ overnight and $50-\mu \mathrm{m}$ thick coronal sections were cut on a vibrating microtome. Sections were stained for PNNs following a pre-embedding immunohistochemistry protocol and preincubated in $1 \% \mathrm{NaBH}_{4}, 3 \%$ hydrogen peroxide, and $10 \%$ normal goat serum before overnight incubation in biotinylated WFA $8 \mu \mathrm{g} / \mathrm{ml}$ (Sigma-Aldrich L1516). Staining was amplified with a Vectastain Elite ABC kit (Vector Laboratories), developed with Ni-DAB and incubated in $1 \%$ osmium tetroxide, then in $1 \%$ uranyl acetate. Then sections were embedded in epoxy resin. Hippocampal pieces were cut from heat-polymerized wafers, glued to a plastic block, cut at $60 \mathrm{~nm}$ with an ultramicrotome, collected on copper grids, and stained with uranyl acetate and Sato's lead. Electron micrographs were imaged on a Philips Tecnai microscope at $80 \mathrm{kV}$.

Electrophysiology. Mice (Charles River Laboratories), age PN 14-18 of either sex, were deeply anesthetized with Fatal-Plus, decapitated, and their brains submerged into oxygenated ice-cold sucrose-substituted artificial CSF (ACSF), pH 7.4, containing the following (in mM): 240 sucrose, $2.0 \mathrm{KCl}, 1 \mathrm{MgCl}_{2}, 2 \mathrm{MgSO}_{4}, 1 \mathrm{CaCl}_{2}, 1.25 \mathrm{NaH}_{2} \mathrm{PO}_{4}, 26 \mathrm{NaHCO}_{3}$ and 10 glucose. Coronal brain slices were cut at $300 \mu \mathrm{m}$ using a vibrating microtome (Leica VT 1000S) and allowed to recover at room temperature in a submersion holding chamber with ACSF containing the following (in mM): $124 \mathrm{NaCl}, 2.5 \mathrm{KCl}, 2 \mathrm{MgCl}_{2}, 2 \mathrm{CaCl}_{2}, 1.25 \mathrm{NaH}_{2} \mathrm{PO}_{4}, 26$ $\mathrm{NaHCO}_{3}$, and 17 D-glucose bubbled with $95 \% \mathrm{O}_{2}$ with $5 \% \mathrm{CO}_{2}$. Slices from one hemisphere were incubated in ACSF and slices from the other in ACSF with $0.05 \mathrm{U} / \mathrm{ml}$ chondroitinase ABC (ChABC; Sigma-Aldrich C3667) for $\geq 2 \mathrm{~h}$ until they were transferred to a recording chamber and continuously bathed (at $2 \mathrm{ml} / \mathrm{min}$ ) in normal ACSF at room temperature (Bukalo et al., 2001). Effectiveness of the chondroitinase treatment was confirmed by staining Amigo2-EGFP slices with WFA as above, except that the $300-\mu \mathrm{m}$-thick slices were cleared using $60 \%$ thiodiethanol in PBS before imaging (Aoyagi et al., 2015).

Whole-cell recordings were made from pyramidal neurons in either the CA2 or CA1 regions. CA2 neurons in the pyramidal layer were initially identified using Amigo2-EGFP mice. Glass borosilicate pipettes 
were filled with a potassium gluconate internal solution containing the following (in mM): 120 K-gluconate, $10 \mathrm{KCl}, 3 \mathrm{MgCl}_{2}, 0.5 \mathrm{EGTA}, 40$ HEPES, $2 \mathrm{Na}_{2}$-ATP, $0.3 \mathrm{Na}-\mathrm{GTP}, \mathrm{pH}$ 7.2. The pipettes had a tip resistance between 2.5 and 4 $\mathrm{M} \Omega$. Baseline synaptic responses were collected for $\geq 5 \mathrm{~min}$. For long-term potentiation (LTP) experiments, a pairing protocol was used. This protocol consisted of $1.5 \mathrm{~min}$ of $3 \mathrm{~Hz}$ presynaptic stimulation (270 pulses) paired with postsynaptic depolarization to $0 \mathrm{mV}$ in voltage-clamp mode. Data were collected using Clampex 10.4 and analyzed using Clampfit software (Molecular Devices). Series and input resistances were monitored by measuring the response to a $10 \mathrm{mV}$ step at each sweep and cells were included for analysis if $<25 \%$ change in series and input resistance was detected. Recordings were not compensated for series resistance.

To determine action potential threshold, whole-cell recordings were performed in current-clamp mode. Current pulses of 180 $\mathrm{ms}$ in $0.2 \mathrm{nA}$ steps were delivered and the membrane potential at which the cells first fired action potentials was measured. To assess excitatory transmission, whole-cell recordings were performed in voltage-clamp mode, and EPSCs were isolated using the $\mathrm{GABA}_{\mathrm{A}}$ receptor antagonist bicuculline (10 $\mu \mathrm{M})$ in the bath solution. EPSC amplitudes were measured at increasing stimulation intensities. Paired-pulse facilitation was assessed under similar conditions.

To measure the NMDA receptor (NMDAR)mediated component of the EPSC, a cesium internal solution was used to block sodiumdependent action potentials, and 5 tetraethylammonium chloride was used for wholecell recordings conducted in the presence of glycine $(5 \mu \mathrm{M})$ and bicuculline $(10 \mu \mathrm{M})$. The cesium internal solution contained the following (in mM): $102 \mathrm{CsOH}, 102$ D-gluconate, 3.7 $\mathrm{NaCl}, 10$ BAPTA, 0.2 EGTA, 20 HEPES, 4 MgATP, $0.3 \mathrm{Na}$-GTP, 5 lidocaine $\mathrm{N}$-ethyl bromide (QX314). EPSCs were measured at -70 and $+40 \mathrm{mV}$ holding potentials. The AMPA receptor (AMPAR)-mediated component of the EPSC was measured $2 \mathrm{~ms}$ after stimulation at $+40 \mathrm{mV}$ (P1), while the NMDAR-component was measured $50 \mathrm{~ms}$ after the same stimulation (P2; Poncer and Malinow, 2001). The AMPAR/ NMDAR ratio was calculated either as a ratio of the $\mathrm{P} 1 / \mathrm{P} 2$ responses at $+40 \mathrm{mV}$ or as a ratio of $\mathrm{P} 1$ at -70 divided by $\mathrm{P} 2$ at $+40 \mathrm{mV}$.

Environmental enrichment. One dam (Amigo2EGFP line) and its litter were singly housed in either standard caging $(32 \times 21 \times 19 \mathrm{~cm})$ or environmental enrichment (EE) caging $(50 \times$ $30 \times 30 \mathrm{~cm}$ ). Standard caging included cotton squares (Enviro-Dri, Shepherd's Specialty Papers) for nesting. EE caging consisted of toys of varying shapes and sizes, such as plastic houses and wooden blocks; extra bedding material, such as cotton squares; sunflower seeds, and fruit/bacon-flavored rodent treats for various forms of sensory stimulation. Objects were repositioned/replaced and treats were replenished every other day. Brains were harvested as a

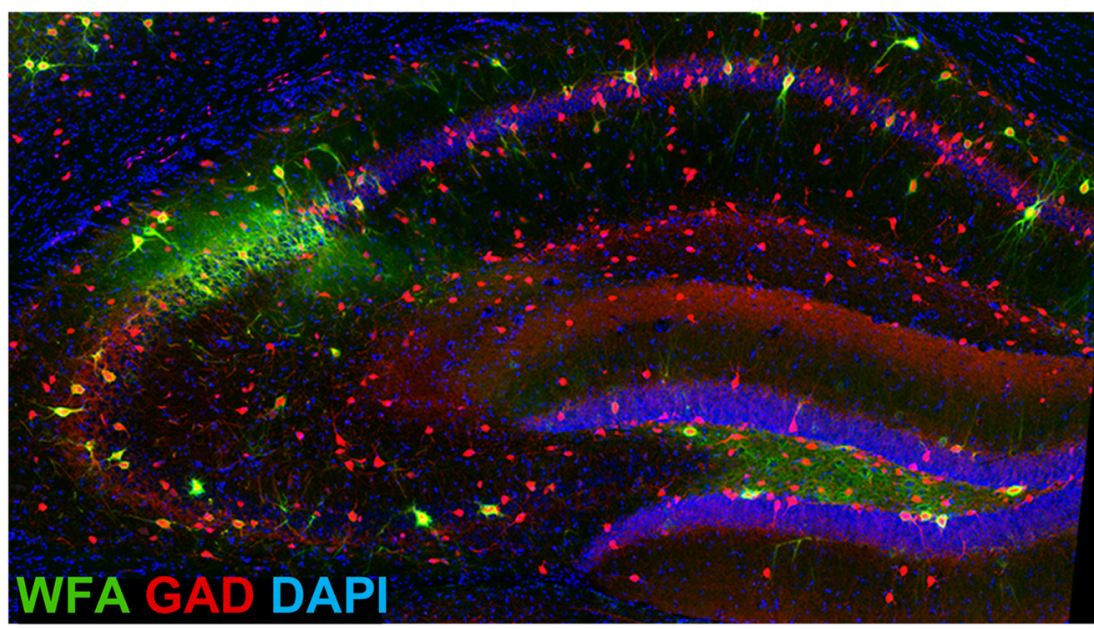

b
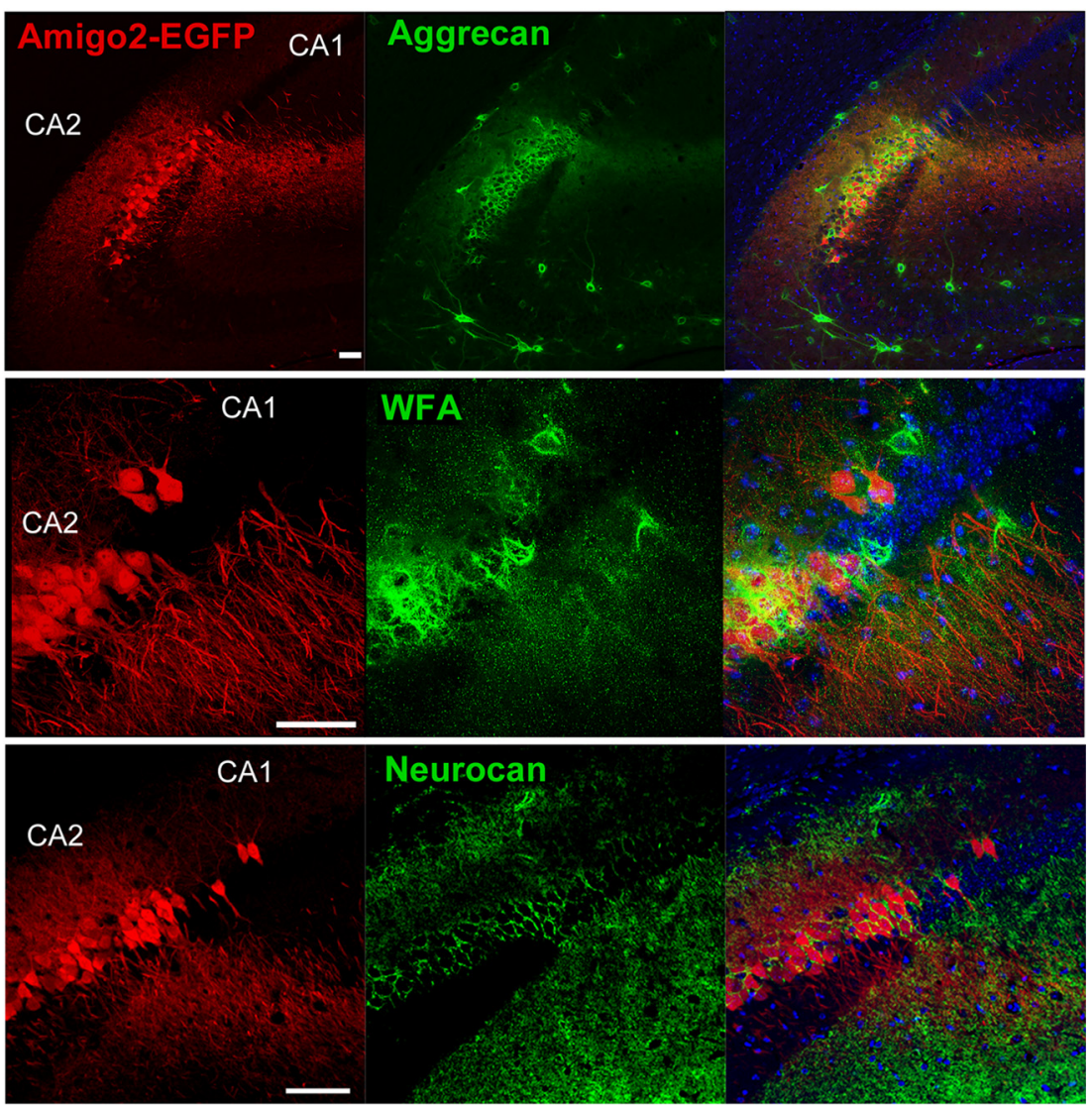

C
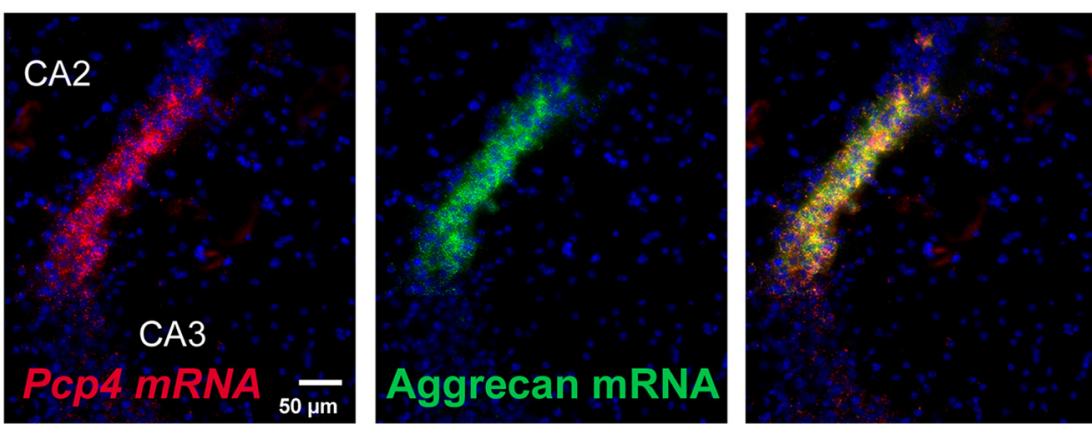

Figure 1. PNN markers surround CA2 pyramidal neurons and dendrites. $\boldsymbol{a}$, Fluorescent labeling of WFA (green) localizes with CA2 pyramidal neurons and with GAD67-positive inhibitory neurons (red) in hippocampus. $\boldsymbol{b}$, Anti-aggrecan (top), WFA (middle), and neurocan (bottom), indicated in green, label EGFP-expressing CA2 pyramidal neurons and their proximal neurites in red (scale bar, $50 \mu \mathrm{m}$ ). c, Fluorescent ISHs showing that aggrecan mRNA (green) and a CA2 marker, Pcp4 mRNA (red), colocalize to the CA2 pyramidal cell layer. Yellow shows the overlapping distribution of these two mRNAs. 
a

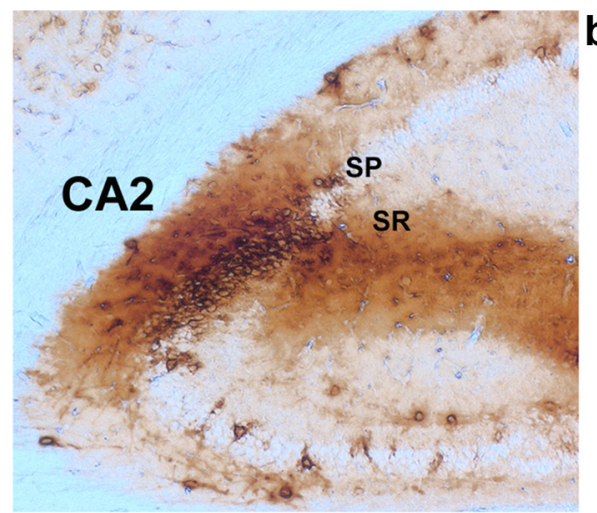

b
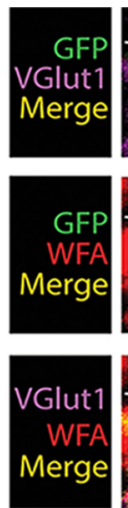
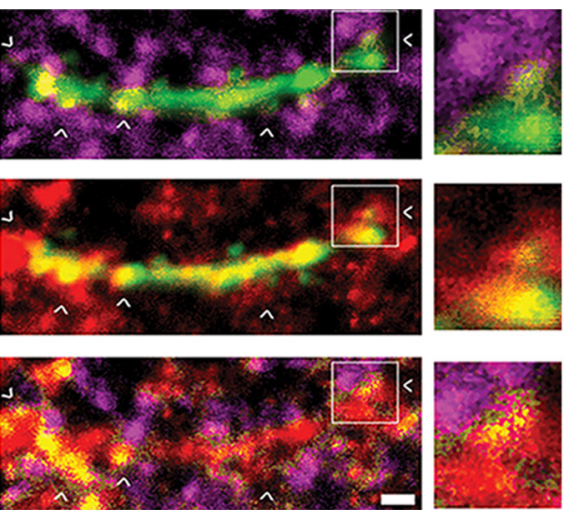

C
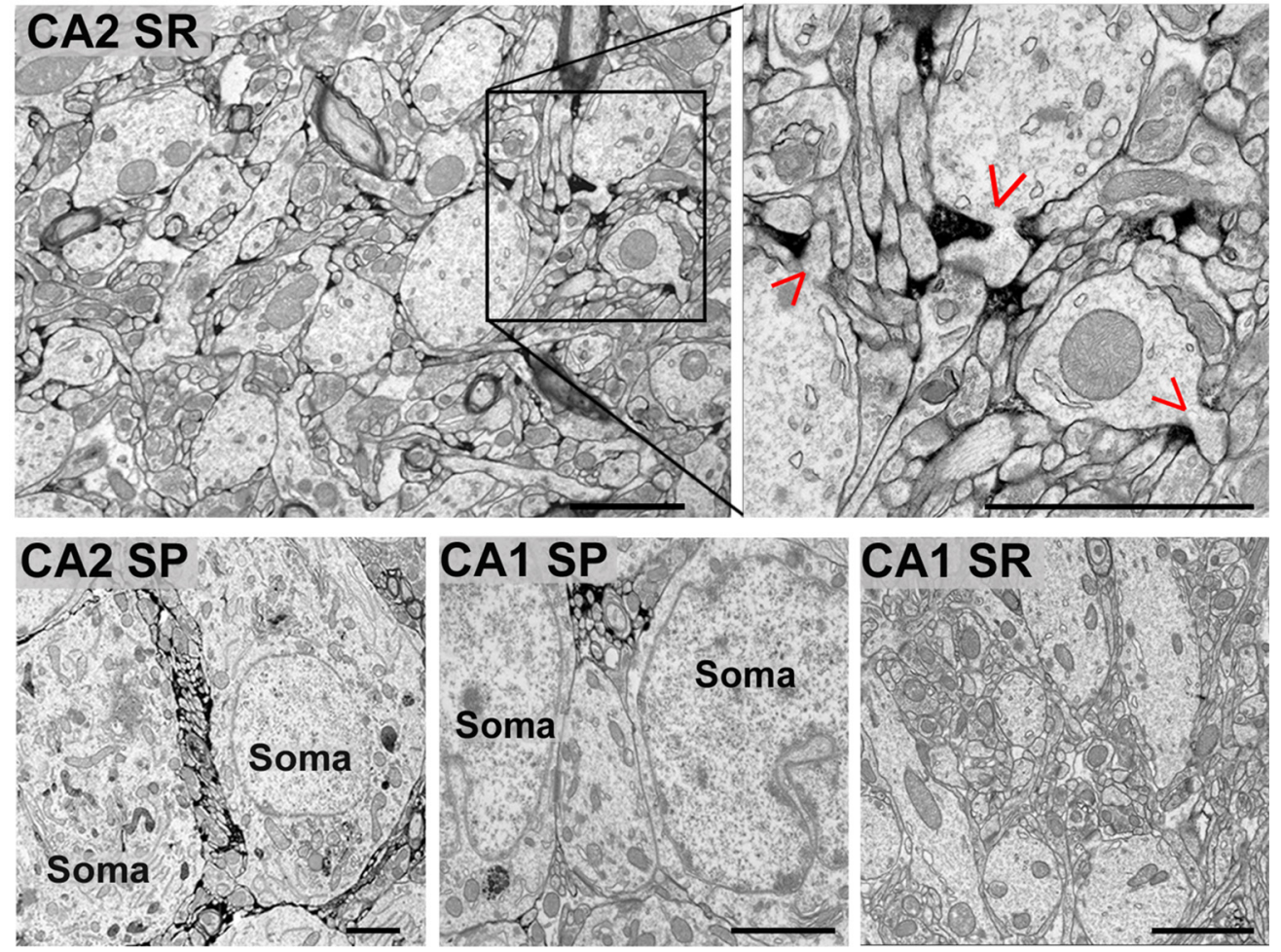

Figure 2. PNN markers are associated with excitatory synapses in area CA2. $\boldsymbol{a}$, WFA immunoperoxidase staining in area CA2 labels CA2 dendrites in the SR in addition to cell bodies in the SP. $\boldsymbol{b}$, WFA label surrounds excitatory synapses on primary apical dendrites of area CA2 SR; green is GFP expressed in neurons in tissue from a thy1-GFP-M mouse, red is WFA, purple is VGLUT1, and yellow shows where two channels depicted in each panel have overlapped (scale bar, $1 \mu \mathrm{m}$ ). $c$, Electron micrographs showing WFA staining along dendritic spines of CA2 pyramidal neurons in the SR (top, red arrowheads), and area CA2 somata in the SP (bottom), and sparse labeling of WFA in area CA1 SP and SR (bottom; scale bar, $2 \mu \mathrm{m}$ ).

described above from male mice at ages PN 14, PN 21, and PN 45 and analyzed for staining of WFA during the $12 \mathrm{~h}$ light cycle. WFA staining intensity was quantified using measures of pixel luminescence value on ImageJ software (National Institutes of Health) using a region of interest (ROI) contoured around either CA2 pyramidal neurons in stratum pyramidale (SP) or CA2 apical dendrites using GFP fluorescence in a dorsal hippocampal section. The mean gray value measure of the image background was subtracted from the mean gray value of the CA2 ROI. Each data point represents one mouse. This study was repeated in a separate cohort of animals and the data combined by normalizing each mean gray value-the value for the PN 45 enriched cohort-for each study. For each experiment, fluorescence quantification was repeated and analyzed with the experimenter blinded to condition. The sample number of mice $(N)$ was $4,5,6,7$, 8 , and 9 for the following groups, respectively: PN 14 standard, PN 14 enriched, PN 45 standard, PN 21 enriched, PN 21 standard, and PN 45 enriched.

In situ hybridization. Adult mouse brains were flash frozen and coronal $20-\mu \mathrm{m}$-thick sections were cut on a cryostat and mounted on
SuperFrost Plus slides (Fisher Scientific 12-550-15). Sections were fixed in $4 \%$ paraformaldehyde for $1 \mathrm{~h}$ at $4^{\circ} \mathrm{C}$, dehydrated in 50,70, and $100 \%$ ethanol, and air-dried at room temperature. Fluorescent RNAscope in situ hybridization (ISH) was performed using an RNAscope Fresh Frozen Multiplex Fluorescent kit according to the manufacturer's protocol to perform target probe hybridization and signal amplification (Advanced Cell Diagnostics). Probes were purchased from Advanced Cell Diagnostics: aggrecan mRNA, mm-acan-C1 (catalog \#300031-C1) and the CA2-marker Purkinje cell protein 4 (PCP4) mRNA, Mm-Pcp4-C2 (catalog \#402311-C2). Fluorescent images were captured on a Zeiss laser-scanning confocal microscope (LSM710).

Statistics. Data in Figures 3 and 4 are expressed as a mean \pm SEM. Data in Figure 3 are expressed as a normalized mean \pm SEM (the enrichment study was repeated once and data were pooled to increase statistical power). Statistical analyses were performed using GraphPad Prism 6.05 software, and significance was calculated using an $\alpha$ level of 0.05 . The Kolmogorov-Smirnov test was used to test normality and variance. 


\section{Results}

PNNs localize to excitatory synapses on CA2 pyramidal

neurons

To confirm our initial impression that PNNs indeed surround CA2 pyramidal neurons and their dendrites, we used tissue from mice expressing EGFP under the promoter of Amigo2, a gene highly expressed in area CA2 (Laeremans et al., 2013). Three different PNN markers, WFA, the chondroitin sulfate proteoglycans aggrecan and neurocan, labeled select inhibitory interneurons throughout the hippocampus (Fig. 1a), but also intensely labeled EGFP-expressing CA2 pyramidal neurons (Fig. $1 b$ ). The origin of PNN matrix components in area CA2 is unknown, so we used fluorescence ISH to determine whether these pyramidal neurons express transcripts for the major PNN component aggrecan. We found that CA2 neurons identified by Purkinje cell protein 4 ( $P C P 4)$ expression, but not CA1 and CA3 neurons, expressed aggrecan mRNA (Fig. 1c). Localization of aggrecan mRNA in the pyramidal cell layer of area CA2 indicates that CA2 pyramidal neurons synthesize at least one major component of the PNN matrix, identifying such neurons as a novel source of PNNs in the hippocampus.

WFA also diffusely labeled the CA2 SR and stratum oriens (Fig. $2 a$ ) near the dendritic spines of excitatory synapses (as defined by label for VGlut1; Fig. 2b). To more precisely characterize this diffuse labeling in the CA2 SR, we turned to electron microscopy. We found that electrondense staining for PNNs appeared around the somata of CA2 pyramidal cells and numerous dendritic spines in the CA2 SR, especially around spine necks and in perisynaptic regions (Fig. 2c). In contrast, staining for PNNs in the neighboring CA1 SP and SR layers was sparse (Fig. 2c).

\section{PNN development is regulated by experience}

Consistent with previous work in other brain regions, including area CA1 (Sur et al., 1988; McRae et al., 2010), we found that PNN staining in CA2 SP and SR increased with age during early postnatal development (Fig. 3a), beginning with minimal WFA fluorescence at PN 14, and increasing in intensity with age. Earlylife sensory deprivation has been demonstrated to delay and attenuate PNN development in several brain regions (Kind et al., 1995; Pizzorusso et al., 2002; McRae et al., 2007). Moreover, in the visual cortex, early-life enrichment has been found to counter effects of dark rearing on PNN development, as well as promote cortical maturation and accelerate the closure of the critical period for plasticity (Bartoletti et al., 2004; Baroncelli et al., 2016). To investigate whether PNNs are similarly modulated by earlylife experience in area CA2, we reared mice in EE conditions. We found that WFA staining intensity in the CA2 SP was significantly greater in EE-exposed animals than those reared in standard housing at PN 21 and $\mathrm{PN} 45(p=0.047$ and $p=0.001$ respec-
PN 21
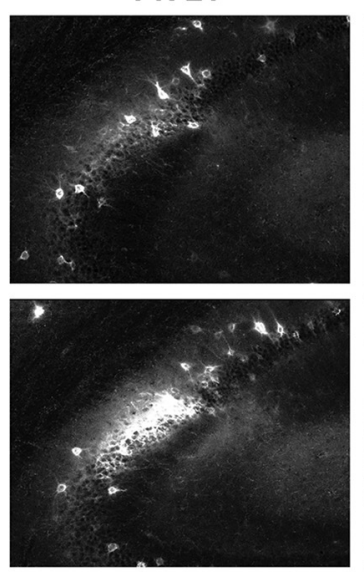

C
PN 45
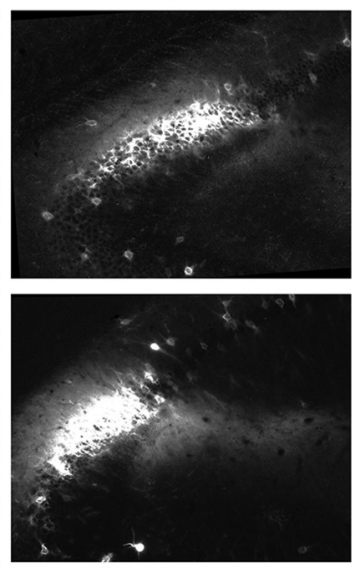

- Standard - Enriched

WFA in Stratum Radiatum

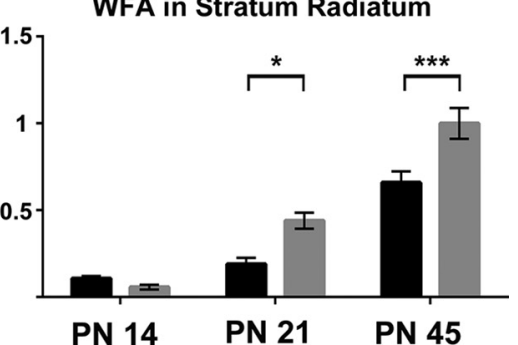

Figure 3. PNNs increase during postnatal development and are increased with experience. $\boldsymbol{a}$, WFA staining in area CA2 is images have been digitally lightened in all panels equally to better display PNNs at PN 14 in area CA2. $\boldsymbol{b}$, Normalized WFA (n) intensity was significantly greater in CA2 SP of EE mice compared with control at PN 21 ( $N=8$ and 7 for control and two-way ANOVA* $\left.{ }^{*}<0.05{ }^{* * *} p=0.0008\right)$. No significant difference was observed at PN $14(N=4$ and 5 for control and $\mathrm{EE}$

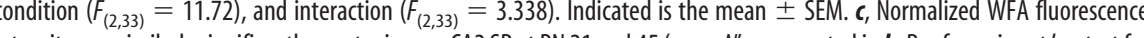
sity was similarly significantly greater in area CA2 SR at PN 21 and 45 (same N's as reported in $\boldsymbol{b}$; Bonferroni post hoc test for pairwise comparison after two-way ANOVA, $\left.{ }^{*} p<0.05,{ }^{* * *} p=0.0009\right)$. A two-way ANOVA for the two conditions at different ages indicated significant main effects of age $\left(F_{(2,33)}=69.55\right)$, condition $\left(F_{(1,33)}=11.93\right)$, and interaction $\left(F_{(2,33)}=3.940\right)$.

tively; two-way ANOVA, Bonferroni's post hoc test for pairwise comparison), but not at PN 14, the earliest age tested (Fig. 3b; $p>$ $0.05)$. WFA staining intensity was similarly significantly greater in the CA2 SR at PN 21 and PN $45(p=0.015$ and $p=0.001$ respectively; two-way ANOVA, Bonferroni's post hoc test for pairwise comparison; Fig. $3 c$ ).

\section{PNNs suppress synaptic plasticity in area CA2}

Excitatory synapses in the CA2 SR fail to express typical LTP (Zhao et al., 2007). To determine whether PNNs act to restrict this type of plasticity in area CA2, we degraded PNNs with the enzyme ChABC in acute hippocampal slices and attempted to induce LTP at CA2 Schaffer collateral synapses. Similar to what has been reported for area CA1 (Bukalo et al., 2001), we found that PNNs are indeed degraded in area CA2 after a $2 \mathrm{~h}$ incubation of slices in ChABC (0.05 $\mu \mathrm{l} / \mathrm{ml}$; Fig. $4 a)$. As reported previously for untreated slices, synaptic responses recorded from CA2 neurons were not potentiated following an LTP "pairing protocol" (92.0 $\pm 0.1 \%$ baseline, $n=10$ ), whereas synaptic responses recorded from CA1 neurons showed typical LTP (140 $\pm 0.1 \%$ baseline, $n=12$; Fig. $4 b$; Zhao et al., 2007). However, a $2 \mathrm{~h}$ incubation with ChABC enabled LTP induction of excitatory synaptic responses in the CA2 SR $(140 \pm 0.2 \%$ baseline, $n=10)$, reaching 
a

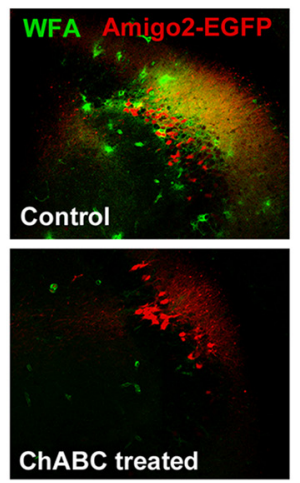

b

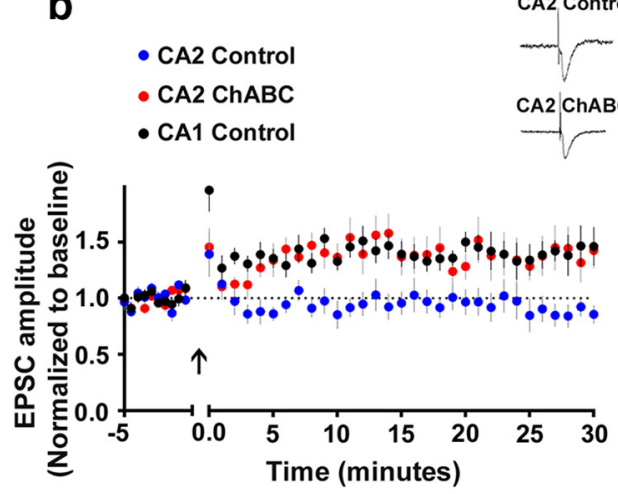

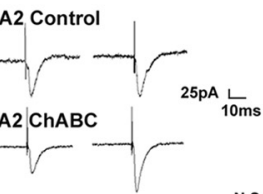

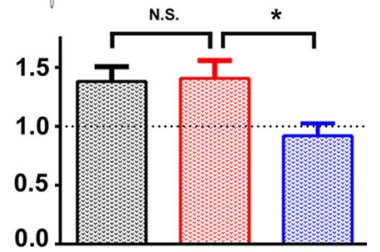

C
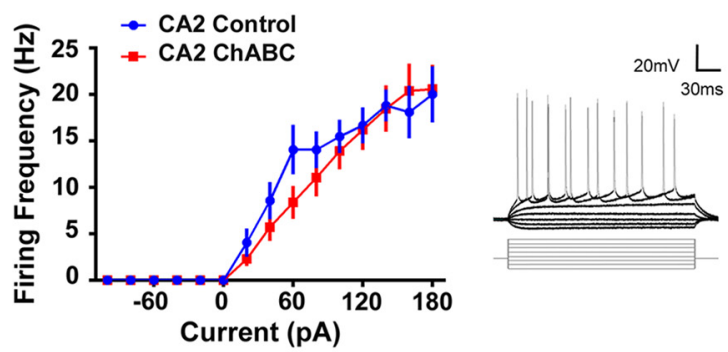

d

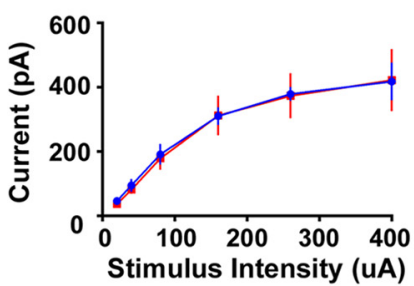

e

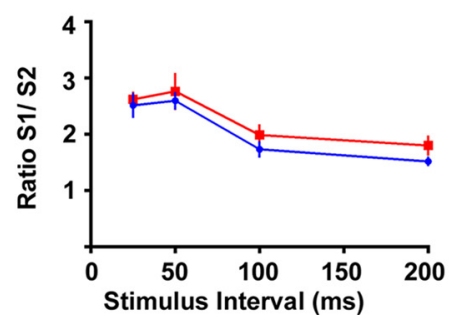

f
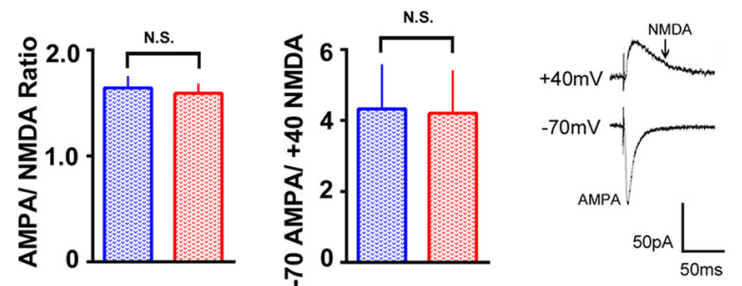

Figure 4. Disruption of PNNs allows for potentiation of EPSCs in CA2 neurons. $\boldsymbol{a}$, PNNs are degraded in 300- $\mu \mathrm{m}$-thick hippocampal slices cleared with thiodiethanol after a $2 \mathrm{~h}$ incubation with ChABC compared with control; fluorescent labeling of PNNs with WFA (green) and CA2 neurons with the Amigo2-EGFP mouse (red). Slices shown are from animals at PN 14. $\boldsymbol{b}$, Plasticity of EPSC amplitudes is enhanced in CA2 neurons treated with the PNN-degrading enzyme ChABC, compared with untreated controls. After baseline, an LTP pairing protocol (270 pulses at $3 \mathrm{~Hz}$ paired with postsynaptic depolarization; at time 0 ), resulted in potentiation of EPSCs in CA2 neurons treated with $0.05 \mathrm{U} / \mathrm{ml}$ ChABC (mean over $22-28 \mathrm{~min}, n=10)$, but not in untreated CA2 controls ( $n=10$ ). One-way ANOVA, Bonferroni's post hoc test for pairwise comparison, ${ }^{*} p<0.05$. Indicated is the mean \pm SEM. LTP induced in CA1 neurons is shown for comparison. Insets are representative traces of EPSCs from CA2 control and CA2 ChABC-treated neurons before and 20 min after the LTP pairing protocol. c, ChABC treatment did not significantly alter action potential firing frequency in response to indicated current injection ( $n=15$ per group; two-way ANOVA, $p>0.05$ ). Example traces show action potential firing of control (untreated) CA2 neuron ( $40 \mathrm{pA}$ current steps from -100 to 180 pA displayed). $\boldsymbol{d}, \boldsymbol{e}, \mathrm{ChABC}$ did not significantly alter excitatory current amplitude ( $n=6$ per group) or paired-pulse ratio in CA2 neurons (S1, peak of first stimulus response; $S 2$, peak of second stimulus response; $n=10 \mathrm{ChABC}$-treated neurons and $n=5$ control; two-way ANOVA, Bonferroni's post hoc test). $f$, ChABC did not significantly alter AMPAR/NMDAR ratio measured at $+40 \mathrm{mV}$ (left) or at $-70 \mathrm{mV}$ ( $n=6 \mathrm{ChABC}$-treated neurons and $n=8$ controls; two-tailed unpaired $t$ test). Measurements in $\boldsymbol{d}-\boldsymbol{f}$ were made in the presence of a GABA $\mathrm{A}_{\mathrm{A}}$ antagonist bicuculline; see Materials and Methods for details. Insets are representative traces of EPSCs from CA2 control holding at -70 and $+40 \mathrm{mV}$.

Table 1. Intrinsic properties of CA2 pyramidal neurons in response to PNN degradation

\begin{tabular}{lllll}
\hline & Intrinsic properties & & & \\
\cline { 2 - 5 } & Resting membrane potential & Membrane capacitance & Neuron input resistance & Decay time \\
\hline CA2 control & $-63.75 \pm 1.05 \mathrm{mV}$ & $143.1 \pm 9.7 \mathrm{pF}$ & $303.5 \pm 10.0 \mathrm{M} \Omega$ & $1.796 \pm 0.088 \mathrm{~ms}$ \\
CA2 ChABC & $-66.17 \pm 0.83 \mathrm{mV}$ & $160.9 \pm 7.9 \mathrm{pF}$ & $259.0 \pm 10.5 \mathrm{M} \Omega^{*}$ & $1.856 \pm 0.064 \mathrm{~ms}$ \\
\hline
\end{tabular}

CA2 neuron input resistance was significantly decreased after $0.05 \mathrm{U} / \mathrm{ml} \mathrm{ChABC}$ treatment $\left(n=11\right.$ per group, ${ }^{*} p<0.0043$, two-tailed unpaired $t$ test). Significant effects were not detected in other properties, including resting membrane potential, membrane capacitance, and decay time. Indicated are the means \pm SEM.

levels comparable to that induced at CA1 synapses $(p=0.02$, two-tailed unpaired $t$ test compared with untreated slices). Similar results were obtained when experiments on ChABC-treated or control-treated slices were performed in the presence of the $\mathrm{GABA}_{\mathrm{A}}$-receptor blocker bicuculline (significant difference between ChABC-treated and untreated slices, $p=0.022$ at $10 \mathrm{~min}$, two-tailed unpaired $t$ test compared with untreated slices, $n=8$ and 4 respectively). Intrinsic properties did not change in a way that could explain this newly uncovered plasticity in area CA2 $(p>0.05$, two-way ANOVA, Bonferroni's post hoc test for pair- wise comparison; Fig. $4 c$; Tables 1, 2). Furthermore, in the presence of bicuculline, we found no significant differences in basal excitatory synaptic transmission (Fig. 4d), paired-pulse facilitation (Fig. 4e), or AMPAR/NMDAR ratio (Fig. 4f). These findings are consistent with a previous report showing that $\mathrm{ChABC}$ treatment had no effect on basal synaptic transmission and pairedpulse facilitation in area CA1. However, in contrast to our work in area CA2, PNN degradation resulted in an attenuation of LTP in area CA1 (Bukalo et al., 2001). These findings indicate that PNNs, or another chondroitinase-sensitive component of the 
Table 2. CA2 action potential firing properties in response to PNN degradation

\begin{tabular}{lllll}
\hline & Action potential & & & \\
\cline { 2 - 5 } & Threshold & Peak amplitude & Decay tau & Rise tau \\
\hline CA2 control & $-47.98 \pm 1.01 \mathrm{mV}$ & $90.48 \pm 2.71 \mathrm{mV}$ & $22.23 \pm 1.52 \mathrm{~ms}$ & $264.3 \pm 16.9 \mathrm{~ms}$ \\
CA2 ChABC & $-47.87 \pm 0.70 \mathrm{mV}$ & $98.9 \pm 4.0 \mathrm{mV}$ & $20.11 \pm 1.26 \mathrm{~ms}$ & $241.0 \pm 19.6 \mathrm{~ms}$ \\
\hline
\end{tabular}

Properties of action potentials in CA2 pyramidal neurons, including threshold, peak amplitude, and decay and rise times (tau), were unchanged after ChABC treatment ( $n=12$ per group, $p>0.05$, two-tailed unpaired $t$ test). Indicated are the means \pm SEM.

ECM, restrict plasticity at the normally plasticity-resistant synapses in CA2 SR.

\section{Discussion}

PNN degradation can reopen critical windows of plasticity in a number of brain regions, but the mechanism of plasticity suppression by PNNs is unclear (Pizzorusso et al., 2002; Gogolla et al., 2009; Romberg et al., 2013). Because PNNs are predominantly associated with inhibitory neurons in the neocortex, it has been suggested that plasticity is altered by a disruption of inhibitory transmission (Dityatev et al., 2010). However, the lack of LTP in area CA2 is unlikely to be due to a disruption of inhibitory transmission, as blockade of GABAergic synaptic transmission is insufficient to restore LTP in area CA2 at excitatory synapses (Zhao et al., 2007; Nasrallah et al., 2015) and was not required for the enabling of LTP reported here. In fact, we present evidence that PNNs play a distinct role in suppressing synaptic potentiation at a population of excitatory synapses on pyramidal neurons, perhaps in addition to any roles PNNs may have related to their expression on inhibitory interneurons. Our findings that PNN degradation enables LTP in area CA2 are in contrast with the observation that PNN degradation modestly disrupts LTP in area CA1 (Bukalo et al., 2001).

PNNs surrounding CA2 neurons are unlikely to be the only factor contributing to the plasticity resistance in this region. CA2 neurons express high levels of the protein regulator of G-protein signaling 14 ( $R G S 14$ ), which has been implicated in the signaling pathway suppressing LTP in area CA2 (Lee et al., 2010). In addition, rodent CA2 neurons have a particularly robust capacity for calcium buffering and extrusion. Raising external calcium levels to $4 \mathrm{~mm}$ is insufficient to induce LTP in area CA2, but the very high concentration of $10 \mathrm{~mm}$ allows for the induction of LTP, indicating that CA2 synapses have the cellular machinery for LTP, but that calcium is limited in the postsynaptic neurons ( $\mathrm{Si}$ mons et al., 2009). Interestingly, both vasopressin and oxytocin, acting through AVPR1b and Oxtr receptors respectively, reinstate ability to induce calcium-dependent LTP in area CA2 (Pagani et al., 2015). Both receptors are known to be coupled to the "Gq"-type G-proteins and therefore can take part in regulating calcium levels in CA2 spines.

PNNs might act through a number of possible plasticitylimiting mechanisms; for example, they may provide a physical barrier for new synaptic contacts, restrict surface receptor mobility, or buffer ions surrounding fast-firing neurons (Morawski et al., 2004; Corvetti and Rossi, 2005; Galtrey et al., 2007; Frischknecht et al., 2009; Kochlamazashvili et al., 2010; Wang and Fawcett, 2012). PNN proteoglycans are highly negatively charged and may function to buffer and restrict diffusion of cations like calcium (Brückner et al., 1993). Together, it might not be coincidental that cation binding to PNNs was found to be saturated at 10 mM (Morawski et al., 2015), a concentration of extracellular calcium that is able to restore plasticity in CA2 neurons (Simons et al., 2009). Alternatively, PNNs may serve to stabilize the postsynaptic density and limit AMPAR insertion into the synapse
(Frischknecht et al., 2009). PNNs have also been linked to neuroprotective functions (Morawski et al., 2004; Cabungcal et al., 2013), and neurons in area CA2 have long been noted as being remarkably resistant to cell death from both seizure and ischemic insult (Sloviter et al., 1991). The role of PNNs in neuroprotection, however, has been less well defined. Therefore, if PNNs act to limit calcium accumulation in CA2 neurons, we would predict that their disruption would lead to damage susceptibility in area CA2 similar to that in areas CA1 and CA3.

We have also identified CA2 pyramidal neurons as a novel source for a major component of PNNs in the hippocampus, suggesting that these excitatory neurons have the capacity to modulate PNN composition throughout development. We show here that PNNs in area CA2 are upregulated by early-life exposure to EE. Interestingly, other studies have reported a decrease in PNNs after exposure to enrichment in adulthood in several brain regions, suggesting that PNNs may be differentially regulated depending on the age that the animal is exposed to enrichment. For example, amblyopic rats exposed to EE in adulthood had reduced PNNs in the visual cortex (Sale et al., 2007); likewise, adult mice exposed to enrichment had reduced PNN density around cerebellar neurons (Foscarin et al., 2011). In contrast, early postnatal enrichment was found to rescue PNN development in dark-reared animals and promote critical period maturation (Bartoletti et al., 2004), similar to the findings in this study. Further investigation into intrinsic factors that modulate PNNs, such as direct manipulation of CA2 activity, or early-life seizure at different developmental stages should provide critical insight into how PNNs in area CA2 may be differentially regulated throughout development (McRae et al., 2010).

Considering that the development of PNNs in hippocampal area CA2 pyramidal neurons parallels the expression timeline of PNNs located in brain regions noted for their critical periods for plasticity, our data raise the intriguing possibility of an early critical period for synaptic plasticity in area CA2. Although the behavioral functions of area CA2 are only beginning to emerge, recent reports implicate area CA2 in social aggression and social recognition memory (Hitti and Siegelbaum, 2014; Pagani et al., 2015; Smith et al., 2016). Developmental regulation of PNNs in area CA2 may therefore represent a therapeutic target for PNNassociated developmental disorders, such as schizophrenia and Rett syndrome (Belichenko et al., 1997; Mauney et al., 2013).

\section{References}

Alpár A, Gärtner U, Härtig W, Brückner G (2006) Distribution of pyramidal cells associated with perineuronal nets in the neocortex of rat. Brain Res 1120:13-22. CrossRef Medline

Aoyagi Y, Kawakami R, Osanai H, Hibi T, Nemoto T (2015) A rapid optical clearing protocol using 2,2' -thiodiethanol for microscopic observation of fixed mouse brain. PloS One 10:e0116280. CrossRef Medline

Baroncelli L, Scali M, Sansevero G, Olimpico F, Manno I, Costa M, Sale A (2016) Experience affects critical period plasticity in the visual cortex through an epigenetic regulation of histone post-translational modifications. J Neurosci 36:3430-3440. CrossRef Medline 
Bartoletti A, Medini P, Berardi N, Maffei L (2004) Environmental enrichment prevents effects of dark-rearing in the rat visual cortex. Nat Neurosci 7:215-216. CrossRef Medline

Belichenko PV, Hagberg B, Dahlström A (1997) Morphological study of neocortical areas in Rett syndrome. Acta Neuropathol 93:50-61. CrossRef Medline

Boulanger LM, Lombroso PJ, Raghunathan A, During MJ, Wahle P, Naegele JR (1995) Cellular and molecular characterization of a brain-enriched protein tyrosine phosphatase. J Neurosci 15:1532-1544. Medline

Brückner G, Brauer K, Härtig W, Wolff JR, Rickmann MJ, Derouiche A, Delpech B, Girard N, Oertel WH, Reichenbach A (1993) Perineuronal nets provide a polyanionic, glia-associated form of microenvironment around certain neurons in many parts of the rat brain. Glia 8:183-200. CrossRef Medline

Bukalo O, Schachner M, Dityatev A (2001) Modification of extracellular matrix by enzymatic removal of chondroitin sulfate and by lack of tenascin- $\mathrm{R}$ differentially affects several forms of synaptic plasticity in the hippocampus. Neuroscience 104:359-369. CrossRef Medline

Cabungcal JH, Steullet P, Morishita H, Kraftsik R, Cuenod M, Hensch TK, Do KQ (2013) Perineuronal nets protect fast-spiking interneurons against oxidative stress. Proc Natl Acad Sci U S A 110:9130-9135. CrossRef Medline

Caruana DA, Alexander GM, Dudek SM (2012) New insights into the regulation of synaptic plasticity from an unexpected place: hippocampal area CA2. Learn Mem 19:391-400. CrossRef Medline

Celio MR (1993) Perineuronal nets of extracellular matrix around parvalbumin-containing neurons of the hippocampus. Hippocampus 3 Spec No:55-60. Medline

Chevaleyre V, Siegelbaum SA (2010) Strong CA2 pyramidal neuron synapses define a powerful disynaptic corticohippocampal loop. Neuron 66: 560-572. CrossRef Medline

Corvetti L, Rossi F (2005) Degradation of chondroitin sulfate proteoglycans induces sprouting of intact Purkinje axons in the cerebellum of the adult rat. J Neurosci 25:7150-7158. CrossRef Medline

Costa C, Tortosa R, Domènech A, Vidal E, Pumarola M, Bassols A (2007) Mapping of aggrecan, hyaluronic acid, heparan sulphate proteoglycans and aquaporin 4 in the central nervous system of the mouse. J Chem Neuroanat 33:111-123. CrossRef Medline

Dityatev A, Brückner G, Dityateva G, Grosche J, Kleene R, Schachner M (2007) Activity-dependent formation and functions of chondroitin sulfate-rich extracellular matrix of perineuronal nets. Dev Neurobiol 67: 570-588. CrossRef Medline

Dityatev A, Schachner M, Sonderegger P (2010) The dual role of the extracellular matrix in synaptic plasticity and homeostasis. Nat Rev Neurosci 11:735-746. CrossRef Medline

Dudek SM, Alexander GM, Farris S (2016) Rediscovering area CA2: unique properties and functions. Nat Rev Neurosci 17:89-102. CrossRef Medline

Feng G, Mellor RH, Bernstein M, Keller-Peck C, Nguyen QT, Wallace M, Nerbonne JM, Lichtman JW, Sanes JR (2000) Imaging neuronal subsets in transgenic mice expressing multiple spectral variants of GFP. Neuron 28:41-51. CrossRef Medline

Foscarin S, Ponchione D, Pajaj E, Leto K, Gawlak M, Wilczynski GM, Rossi F, Carulli D (2011) Experience-dependent plasticity and modulation of growth regulatory molecules at central synapses. PloS One 6:e16666. CrossRef Medline

Frischknecht R, Heine M, Perrais D, Seidenbecher CI, Choquet D, Gundelfinger ED (2009) Brain extracellular matrix affects AMPA receptor lateral mobility and short-term synaptic plasticity. Nat Neurosci 12:897904. CrossRef Medline

Fuxe K, Tinner B, Staines W, David G, Agnati LF (1997) Regional distribution of neural cell adhesion molecule immunoreactivity in the adult rat telencephalon and diencephalon. Partial colocalization with heparan sulfate proteoglycan immunoreactivity. Brain Res 746:25-33. CrossRef Medline

Galtrey CM, Asher RA, Nothias F, Fawcett JW (2007) Promoting plasticity in the spinal cord with chondroitinase improves functional recovery after peripheral nerve repair. Brain 130:926-939. Medline

Gogolla N, Caroni P, Lüthi A, Herry C (2009) Perineuronal nets protect fear memories from erasure. Science 325:1258-1261. CrossRef Medline

Guimarães A, Zaremba S, Hockfield S (1990) Molecular and morphological changes in the cat lateral geniculate nucleus and visual cortex induced by visual deprivation are revealed by monoclonal antibodies Cat-304 and Cat-301. J Neurosci 10:3014-3024. Medline

Hensch TK (2004) Critical period regulation. Annu Rev Neurosci 27:549579. CrossRef Medline

Hitti FL, Siegelbaum SA (2014) The hippocampal CA2 region is essential for social memory. Nature 508:88-92. CrossRef Medline

Horii-Hayashi N, Sasagawa T, Matsunaga W, Nishi M (2015) Development and structural variety of the chondroitin sulfate proteoglycans-contained extracellular matrix in the mouse brain. Neural Plast 2015:256389. CrossRef Medline

Kalb RG, Hockfield S (1988) Molecular evidence for early activitydependent development of hamster motor neurons. J Neurosci 8:2350 2360. Medline

Kind PC, Beaver CJ, Mitchell DE (1995) Effects of early periods of monocular deprivation and reverse lid suture on the development of Cat-301 immunoreactivity in the dorsal lateral geniculate nucleus (dLGN) of the cat. J Comp Neurol 359:523-536. CrossRef Medline

Kochlamazashvili G, Henneberger C, Bukalo O, Dvoretskova E, Senkov O, Lievens PM, Westenbroek R, Engel AK, Catterall WA, Rusakov DA, Schachner M, Dityatev A (2010) The extracellular matrix molecule hyaluronic acid regulates hippocampal synaptic plasticity by modulating L-type $\mathrm{Ca}(2+)$ channels. Neuron 67:116-128. CrossRef Medline

Kohara K, Pignatelli M, Rivest AJ, Jung HY, Kitamura T, Suh J, Frank D, Kajikawa K, Mise N, Obata Y, Wickersham IR, Tonegawa S (2014) Cell type-specific genetic and optogenetic tools reveal hippocampal CA2 circuits. Nat Neurosci 17:269-279. CrossRef Medline

Laeremans A, Nys J, Luyten W, D’Hooge R, Paulussen M, Arckens L (2013) AMIGO2 mRNA expression in hippocampal CA2 and CA3a. Brain Struct Funct 218:123-130. CrossRef Medline

Lander C, Kind P, Maleski M, Hockfield S (1997) A family of activitydependent neuronal cell-surface chondroitin sulfate proteoglycans in cat visual cortex. J Neurosci 17:1928-1939. Medline

Lee SE, Simons SB, Heldt SA, Zhao M, Schroeder JP, Vellano CP, Cowan DP, Ramineni S, Yates CK, Feng Y, Smith Y, Sweatt JD, Weinshenker D, Ressler KJ, Dudek SM, Hepler JR (2010) RGS14 is a natural suppressor of both synaptic plasticity in CA2 neurons and hippocampal-based learning and memory. Proc Natl Acad Sci U S A 107:16994-16998. CrossRef Medline

Mauney SA, Athanas KM, Pantazopoulos H, Shaskan N, Passeri E, Berretta S, Woo TU (2013) Developmental pattern of perineuronal nets in the human prefrontal cortex and their deficit in schizophrenia. Biol Psychiatry 74:427-435. CrossRef Medline

McRae PA, Rocco MM, Kelly G, Brumberg JC, Matthews RT (2007) Sensory deprivation alters aggrecan and perineuronal net expression in the mouse barrel cortex. J Neurosci 27:5405-5413. CrossRef Medline

McRae PA, Baranov E, Sarode S, Brooks-Kayal AR, Porter BE (2010) Aggrecan expression, a component of the inhibitory interneuron perineuronal net, is altered following an early-life seizure. Neurobiol Dis 39:439-448. CrossRef Medline

Morawski M, Brückner MK, Riederer P, Brückner G, Arendt T (2004) Perineuronal nets potentially protect against oxidative stress. Exp Neurol 188:309-315. CrossRef Medline

Morawski M, Reinert T, Meyer-Klaucke W, Wagner FE, Tröger W, Reinert A, Jäger C, Brückner G, Arendt T (2015) Ion exchanger in the brain: quantitative analysis of perineuronally fixed anionic binding sites suggests diffusion barriers with ion sorting properties. Sci Rep 5:16471. CrossRef Medline

Nasrallah K, Piskorowski RA, Chevaleyre V (2015) Inhibitory plasticity permits the recruitment of CA2 pyramidal neurons by CA3 $(1,2,3)$. eNeuro 2 pii:ENEURO.0049-15.2015. CrossRef Medline

Pagani JH, Zhao M, Cui Z, Avram SK, Caruana DA, Dudek SM, Young WS (2015) Role of the vasopressin $1 \mathrm{~b}$ receptor in rodent aggressive behavior and synaptic plasticity in hippocampal area CA2. Mol Psychiatry 20:490499. CrossRef Medline

Pelkey KA, Askalan R, Paul S, Kalia LV, Nguyen TH, Pitcher GM, Salter MW, Lombroso PJ (2002) Tyrosine phosphatase STEP is a tonic brake on induction of long-term potentiation. Neuron 34:127-138. CrossRef Medline

Pizzorusso T, Medini P, Berardi N, Chierzi S, Fawcett JW, Maffei L (2002) Reactivation of ocular dominance plasticity in the adult visual cortex. Science 298:1248-1251. CrossRef Medline 
Poncer JC, Malinow R (2001) Postsynaptic conversion of silent synapses during LTP affects synaptic gain and transmission dynamics. Nat Neurosci 4:989-996. CrossRef Medline

Romberg C, Yang S, Melani R, Andrews MR, Horner AE, Spillantini MG, Bussey TJ, Fawcett JW, Pizzorusso T, Saksida LM (2013) Depletion of perineuronal nets enhances recognition memory and long-term depression in the perirhinal cortex. J Neurosci 33:7057-7065. CrossRef Medline

Sale A, Maya Vetencourt JF, Medini P, Cenni MC, Baroncelli L, De Pasquale R, Maffei L (2007) Environmental enrichment in adulthood promotes amblyopia recovery through a reduction of intracortical inhibition. Nat Neurosci 10:679-681. CrossRef Medline

Simons SB, Escobedo Y, Yasuda R, Dudek SM (2009) Regional differences in hippocampal calcium handling provide a cellular mechanism for limiting plasticity. Proc Natl Acad Sci U S A 106:14080-14084. CrossRef Medline
Sloviter RS, Sollas AL, Barbaro NM, Laxer KD (1991) Calcium-binding protein (calbindin-D28K) and parvalbumin immunocytochemistry in the normal and epileptic human hippocampus. J Comp Neurol 308:381-396. CrossRef Medline

Smith AS, Williams Avram SK, Cymerblit-Sabba A, Song J, Young WS (2016) Targeted activation of the hippocampal CA2 area strongly enhances social memory. Mol Psychiatry. Advance online publication. Retrieved May 6, 2016. CrossRef Medline

Sur M, Frost DO, Hockfield S (1988) Expression of a surface-associated antigen on Y-cells in the cat lateral geniculate nucleus is regulated by visual experience. J Neurosci 8:874-882. Medline

Wang D, Fawcett J (2012) The perineuronal net and the control of CNS plasticity. Cell Tissue Res 349:147-160. CrossRef Medline

Zhao M, Choi YS, Obrietan K, Dudek SM (2007) Synaptic plasticity (and the lack thereof) in hippocampal CA2 neurons. J Neurosci 27:1202512032. CrossRef Medline 